\title{
Verification of Convergence Rates of Numerical Solutions for Parabolic Equations
}

\author{
Darae Jeong $\mathbb{D}^{1},{ }^{1}$ Yibao Li, ${ }^{2}$ Chaeyoung Lee, ${ }^{3}$ Junxiang Yang, ${ }^{3}$ \\ Yongho Choi $\left(1,{ }^{4}\right.$ and Junseok Kim ${ }^{3}{ }^{3}$ \\ ${ }^{1}$ Department of Mathematics, Kangwon National University, Gangwon-do 24341, Republic of Korea \\ ${ }^{2}$ School of Mathematics and Statistics, Xian Jiaotong University, Xian 710049, China \\ ${ }^{3}$ Department of Mathematics, Korea University, Seoul 02841, Republic of Korea \\ ${ }^{4}$ Department of Mathematics and Big Data, Daegu University, Gyeongsan-si, Gyeongsangbuk-do 38453, Republic of Korea
}

Correspondence should be addressed to Junseok Kim; cfdkim@korea.ac.kr

Received 19 February 2019; Revised 11 May 2019; Accepted 4 June 2019; Published 23 June 2019

Academic Editor: Nicolae Herisanu

Copyright (C) 2019 Darae Jeong et al. This is an open access article distributed under the Creative Commons Attribution License, which permits unrestricted use, distribution, and reproduction in any medium, provided the original work is properly cited.

In this paper, we propose a verification method for the convergence rates of the numerical solutions for parabolic equations. Specifically, we consider the numerical convergence rates of the heat equation, the Allen-Cahn equation, and the Cahn-Hilliard equation. Convergence test results show that if we refine the spatial and temporal steps at the same time, then we have the secondorder convergence rate for the second-order scheme. However, in the case of the first-order in time and the second-order in space scheme, we may have the first-order or the second-order convergence rates depending on starting spatial and temporal step sizes. Therefore, for a rigorous numerical convergence test, we need to perform the spatial and the temporal convergence tests separately.

\section{Introduction}

Many numerical schemes for differential equations have been developed and tested in terms of convergence rates. If an analytic solution is available, then we can use it as a reference solution for the convergence test. Otherwise, we can use a reference solution obtained from a numerical solution with very fine space and time step sizes. Some scientific journal considers manuscripts only if accuracy and convergence of numerical solutions are established by discussion of results on multiple grids. Recently, there have been many research studies on the second-order convergence schemes for parabolictype partial differential equations [1-12]. To demonstrate the second-order convergence, some authors [1-3] showed the convergence by refining the spatial and temporal steps at the same time; some authors in [4-10] showed the convergence by refining the spatial and temporal steps separately.

In this work, we will show that we may have the secondorder convergence even though the numerical scheme is the first-order accurate in time and the second-order accurate in space if we refine the spatial and temporal steps at the same time. Therefore, for a rigorous numerical convergence test, we need to perform the spatial and the temporal convergence tests separately. We validate these claims from the convergence rates of the numerical solutions for parabolic equations. Specifically, we consider the convergence rates of the numerical schemes for the heat equation, the Allen-Cahn (AC) equation, and the Cahn-Hilliard (CH) equation, which have been studied theoretically by many researchers in recent years [14-20].

The first equation is the heat equation on $\Omega=(0,2 \pi)$ :

$$
\frac{\partial \phi(x, t)}{\partial t}=\frac{\partial^{2} \phi(x, t)}{\partial x^{2}} \text { for } t>0
$$

with the zero Neumann boundary condition $\phi_{x}(0, t)=$ $\phi_{x}(2 \pi, t)=0$. $(0,1)$ :

The second equation is the $\mathrm{AC}$ equation $[22,23]$ on $\Omega=$

$$
\frac{\partial \phi(x, t)}{\partial t}=\frac{F^{\prime}(\phi(x, t))}{\epsilon^{2}}+\Delta \phi(x, t) \quad \text { for } t>0
$$




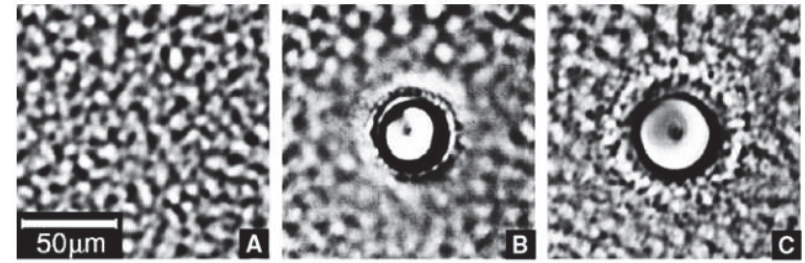

(a)

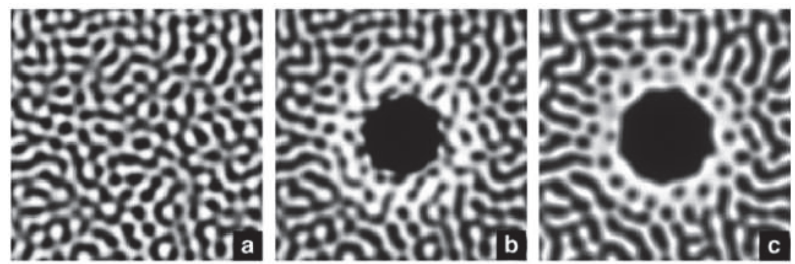

(b)

Figure 1: Phase separation in a binary mixture by (a) experiment and (b) numerical tests. Adapted from Voit et al. [13] with the permission of American Physical Society.

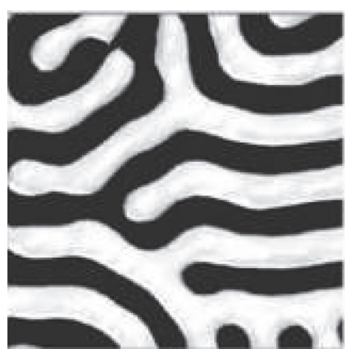

(a)

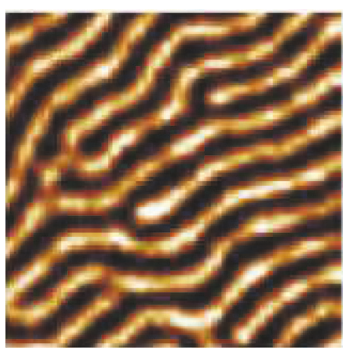

(e)

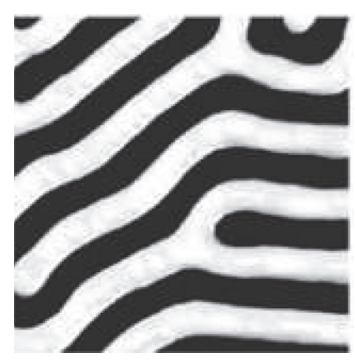

(b)

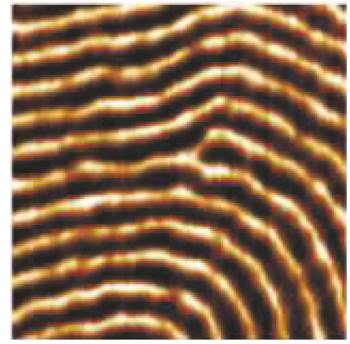

(f)

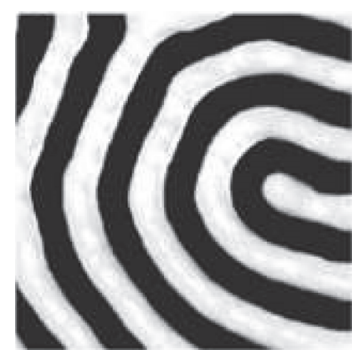

(c)

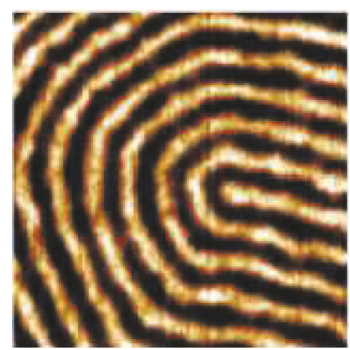

(g)

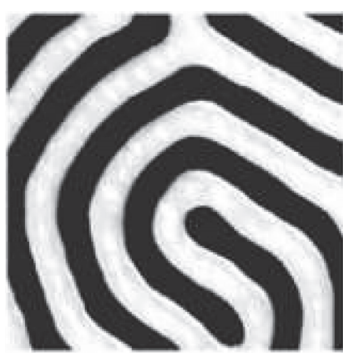

(d)

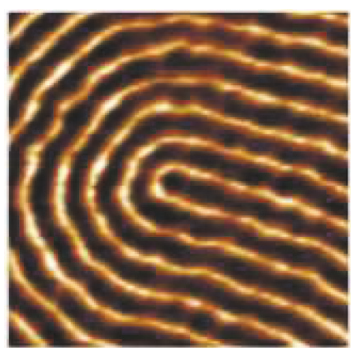

(h)

FIGURE 2: Formation of block copolymer by numerical (first row) and numerical (second row) tests. Adapted from Horvat et al. [21] with the permission of American Chemical Society.

with the periodic boundary condition $\phi(0, t)=\phi(1, t)$. The phase-field $\phi(x, t)$ is the difference between the concentrations of the two mixtures' components, $F(\phi)=0.25\left(\phi^{2}-1\right)^{2}$, and $\epsilon$ is a positive constant. The $\mathrm{AC}$ equation has a wide range of applications such as mean curvature flows [24-26], two-phase incompressible fluids [27], complex dynamics of dendritic growth [28, 29], image inpainting [30], and image segmentation $[31,32]$.

The third equation is the $\mathrm{CH}$ equation [33] on $\Omega=(0,1)$ :

$$
\begin{aligned}
\frac{\partial \phi}{\partial t}(x, t) & =\Delta \mu(x, t) \quad \text { for } t>0, \\
\mu(x, t) & =F^{\prime}(\phi(x, t))-\epsilon^{2} \Delta \phi(x, t),
\end{aligned}
$$

with the periodic boundary condition

$$
\begin{aligned}
& \phi(0, t)=\phi(1, t), \\
& \mu(0, t)=\mu(1, t) .
\end{aligned}
$$

This $\mathrm{CH}$ equation is widely used in applications such as phase separation [34], topology optimization [35], multiphase incompressible fluid flows [36-39], image inpainting [40], surface reconstruction [41], diblock copolymer [42], tumor growth simulation [43], and microstructures with elastic inhomogeneity [44].

Some studies suggest a mathematical model as a way to reproduce the experiment and present a numerical solution of it, as shown in Figures 1 and 2. Figure 1 shows the experimental and numerical pattern formations of binary mixture [13].

Other example [21] is the formation of block copolymer; the numerical and experimental results are shown in first and second rows in Figure 2, respectively.

Along with the study of mathematical model, various numerical schemes have been developed as a way to solve this accurately. At this time, a convergence test can be performed as a method for verifying the accuracy of such a numerical solution, which must be performed correctly. 
Therefore, we present the right method validating the accuracy order of the numerical solution for parabolic-type equations with various benchmark tests.

This paper is structured in the following manner. In Section 2, we describe the numerical solution algorithms for the three equations. In Section 3, we present several numerical results. Then, in Section 4, we conclude.

\section{Numerical Solution}

In this section, we present the numerical solutions for the three equations by a finite difference method [45].

2.1. Heat Equation. First, we consider the heat equation. Let $\Omega=(0,2 \pi)$ be discretized by using a uniform grid with $h=$ $2 \pi / N_{x}$, where $N_{x}$ is the number of subintervals (see Figure 3 ).

Let $\phi_{i}^{n}$ be approximation of $\phi\left(x_{i}, t_{n}\right)$, where $x_{i}=(i-0.5) h$, $t_{n}=n \Delta t$, and $\Delta t$ is the temporal step size. Now, we apply a $\theta$ method to the heat (1) as follows:

$$
\frac{\phi_{i}^{n+1}-\phi_{i}^{n}}{\Delta t}=(1-\theta) \Delta_{h} \phi_{i}^{n}+\theta \Delta_{h} \phi_{i}^{n+1},
$$

for $1 \leq i \leq N_{x}$,

where $\Delta_{h} \phi_{i}^{n}=\left(\phi_{i-1}^{n}-2 \phi_{i}^{n}+\phi_{i+1}^{n}\right) / h^{2}$ and $0 \leq \theta \leq 1$. For the homogeneous Neumann boundary condition, we set $\phi_{0}^{n}=\phi_{1}^{n}$ and $\phi_{N_{x}+1}^{n}=\phi_{N_{x}}^{n}$ for all $n=0,1, \ldots$ If $\theta=0.5$, then (6) becomes the Crank-Nicolson (CN) scheme [46] and the convergence rate is $O\left(\Delta t^{2}\right)+O\left(h^{2}\right)$. If $\theta=1$, then (6) becomes a fully implicit scheme with the convergence rate, $O(\Delta t)+$ $O\left(h^{2}\right)$. Here, (6) is solved by using the Thomas algorithm [45]. Let the error be defined as $\mathbf{e}_{N_{x}}^{N_{t}}=\left(e_{1}^{N_{t}}, e_{2}^{N_{t}}, \ldots, e_{N_{x}}^{N_{t}}\right)$, where $e_{i}^{N_{t}}=\phi_{i}^{N_{t}}-\phi\left(x_{i}, t_{N_{t}}\right)$ for $i=1, \ldots, N_{x}$ and let $\left\|\mathbf{e}_{N_{x}}^{N_{t}}\right\|_{2}=$ $\sqrt{\left(1 / N_{x}\right) \sum_{i=1}^{N_{x}}\left(e_{i}^{N_{t}}\right)^{2}}$.

2.2. AC Equation. We consider the numerical solution for the AC equation. Let $\phi_{i}^{n}$ be approximation of $\phi\left(x_{i}, t_{n}\right)$, where $x_{i}=$ $i h$ and $h=1 / N_{x}$. We discretize (2) by applying the $\theta$-method as follows:

$$
\begin{aligned}
\frac{\phi_{i}^{n+1}-\phi_{i}^{n}}{\Delta t}= & (1-\theta)\left(-\frac{F^{\prime}\left(\phi_{i}^{n}\right)}{\epsilon^{2}}+\Delta_{h} \phi_{i}^{n}\right) \\
& +\theta\left(-\frac{F^{\prime}\left(\phi_{i}^{n+1}\right)}{\epsilon^{2}}+\Delta_{h} \phi_{i}^{n+1}\right),
\end{aligned}
$$

where $0 \leq \theta \leq 1$ and $0 \leq i \leq N_{x}$. For the periodic boundary condition, we set $\phi_{0}^{n}=\phi_{N_{x}}^{n}$ for all $n=0,1, \ldots$ Here, we use a multigrid algorithm [47-49] to solve the discrete (7).

2.3. CH Equation. In this problem, we apply the unconditionally gradient stable method [50] to (3) and (4):

$$
\begin{gathered}
\frac{\phi_{i}^{n+1}-\phi_{i}^{n}}{\Delta t}=\Delta_{h} \mu_{i}^{n+1}, \quad \text { for } 0 \leq i \leq N_{x}, \\
\mu_{i}^{n+1}=\left(\phi_{i}^{n+1}\right)^{3}-\phi_{i}^{n}-\epsilon^{2} \Delta \phi_{i}^{n+1}
\end{gathered}
$$

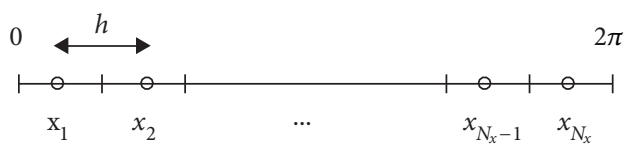

Figure 3: Uniform grid with a spatial step size $h$.

with the periodic boundary condition. Note that this scheme is first-order in time and second-order in space. To solve the discrete equation (8) and (9), we also use a multigrid algorithm [47-49].

\section{Numerical Experiments}

3.1. Heat Equation. For numercial test, the initial state is given by $\phi(x, 0)=\cos (x)$ on $\Omega=(0,2 \pi)$. Therefore, the closed-form solution of (1) is $\phi(x, t)=e^{-t} \cos (x)$. Now, we compute the discrete $l_{2}$-norm error at $T=0.1$.

3.1.1. Convergence Test for the CN Scheme. Table 1 lists the $l_{2}$-norm error and temporal convergence rates for the $\mathrm{CN}$ scheme with various $\Delta t=T / N_{t}$ and the fixed space step size $h=2 \pi / N_{x}$. Here, the temporal convergence rate is defined as $\log _{2}\left(\left\|\mathbf{e}_{N_{x}}^{N_{t}}\right\|_{2} /\left\|\mathbf{e}_{N_{x}}^{2 N_{t}}\right\|_{2}\right)$. The boxed rates are the numbers greater than or equal to 1.9. As we refine the space grid size, we have more second-order temporal convergence results.

Table 2 lists the $l_{2}$-norm error and spatial convergence rates for the $\mathrm{CN}$ scheme with various $h=2 \pi / N_{x}$ and the fixed time step size $\Delta t=T / N_{t}$. The spatial convergence rate is defined as $\log _{2}\left(\left\|\mathbf{e}_{N_{x}}^{N_{t}}\right\|_{2} /\left\|\mathbf{e}_{2 N_{x}}\right\|_{2}\right)$. As we refine the time grid size, we have more second-order spatial convergence results.

Table 3 lists the $l_{2}$-norm error and convergence rates for the CN scheme with various $h=2 \pi / N_{x}$ and $\Delta t=T / N_{t}$. The convergence rate is defined as $\log _{2}\left(\left\|\mathbf{e}_{N_{x}}^{N_{t}}\right\|_{2} /\left\|\mathbf{e}_{2 N_{x}}^{2 N_{t}}\right\|_{2}\right)$. We have second-order convergence results for all the cases. We have a sequence of the second-order convergence (diagonal sequence in Table 3) regardless of starting pair, $\left(N_{x}, N_{t}\right)$. We can find some examples of this approach. In [1], the refinement path was taken to be $\Delta t=0.2 h / \sqrt{2}$ to show the second-order convergence of the proposed numerical scheme for the Cahn-Hilliard-Navier-Stokes equation. In [2], $\Delta t=h$ was taken to show the second-order convergence for solving space fractional diffusion equations. In [3], $\Delta t=h$ was used to show the second-order convergence rate for a two-sided space-fractional diffusion equation with variable coefficients.

We have the following relation between errors:

$$
\begin{aligned}
\log _{2} \frac{\left\|\mathbf{e}_{N_{x}}^{N_{t}}\right\|_{2}}{\left\|\mathbf{e}_{2 N_{x}}^{2 N_{t}}\right\|_{2}} & =\log _{2} \frac{\left\|\mathbf{e}_{N_{x}}\right\|_{2}}{\left\|\mathbf{e}_{N_{x}}^{2 N_{t}}\right\|_{2}}+\log _{2} \frac{\left\|\mathbf{e}_{N_{x}}^{2 N_{t}}\right\|_{2}}{\left\|\mathbf{e}_{2 N_{x}}^{22 N_{x}}\right\|_{2}} \\
& =\log _{2} \frac{\left\|\mathbf{e}_{N_{x}}^{N_{t}}\right\|_{2}}{\left\|\mathbf{e}_{2 N_{x}}^{N_{t}}\right\|_{2}}+\log _{2} \frac{\left\|\mathbf{e}_{2 N_{x}}^{N_{t}}\right\|_{2}}{\left\|\mathbf{e}_{2 N_{x}}^{2 N_{t}}\right\|_{2}} .
\end{aligned}
$$


TABLE 1: $l_{2}$-norm error and temporal convergence rates for the CN scheme with various $\Delta t$ at $T=0.1$.

\begin{tabular}{|c|c|c|c|c|c|}
\hline$N_{t} \backslash N_{x}$ & 100 & 200 & 400 & 800 & 1600 \\
\hline 1 & $3.23 e-5$ & $4.81 e-5$ & $5.21 \mathrm{e}-5$ & $5.31 \mathrm{e}-5$ & $5.33 e-5$ \\
\hline rate & 2.06 & 2.58 & 2.12 & \begin{tabular}{|l|}
2.03 \\
\end{tabular} & \begin{tabular}{|l|}
2.01 \\
\end{tabular} \\
\hline 2 & $7.73 e-6$ & 8.07e-6 & $1.20 \mathrm{e}-5$ & $1.30 \mathrm{e}-5$ & $1.33 \mathrm{e}-5$ \\
\hline rate & -1.20 & 2.06 & 2.57 & \begin{tabular}{|l|}
2.11 \\
\end{tabular} & 2.03 \\
\hline 4 & $1.77 \mathrm{e}-5$ & $1.93 \mathrm{e}-6$ & $2.02 \mathrm{e}-6$ & $3.00 \mathrm{e}-6$ & $3.25 \mathrm{e}-6$ \\
\hline rate & -0.19 & -1.20 & 2.06 & 2.57 & 2.11 \\
\hline 8 & $2.02 \mathrm{e}-5$ & $4.43 e-6$ & $4.82 \mathrm{e}-7$ & $5.04 \mathrm{e}-7$ & $7.51 \mathrm{e}-7$ \\
\hline rate & -0.04 & -0.19 & -1.20 & 2.06 & 2.57 \\
\hline 16 & $2.08 e-5$ & $5.05 e-6$ & $1.11 \mathrm{e}-6$ & $1.21 \mathrm{e}-7$ & $1.26 \mathrm{e}-7$ \\
\hline rate & -0.01 & -0.04 & -0.19 & -1.20 & 2.06 \\
\hline 32 & $2.10 \mathrm{e}-5$ & $5.21 \mathrm{e}-6$ & $1.26 \mathrm{e}-6$ & $2.77 e-7$ & $3.02 \mathrm{e}-8$ \\
\hline rate & -0.00 & -0.01 & -0.04 & -0.19 & -1.20 \\
\hline 64 & $2.10 \mathrm{e}-5$ & $5.25 e-6$ & $1.30 e-6$ & $3.16 \mathrm{e}-7$ & $6.92 \mathrm{e}-8$ \\
\hline rate & -0.00 & -0.00 & -0.01 & -0.04 & -0.19 \\
\hline 128 & $2.10 \mathrm{e}-5$ & $5.26 e-6$ & $1.31 e-6$ & $3.26 \mathrm{e}-7$ & $7.90 \mathrm{e}-8$ \\
\hline rate & -0.00 & -0.00 & -0.00 & -0.01 & -0.04 \\
\hline 256 & $2.10 e-5$ & $5.26 \mathrm{e}-6$ & $1.31 \mathrm{e}-6$ & $3.28 \mathrm{e}-7$ & $8.14 \mathrm{e}-8$ \\
\hline rate & -0.00 & -0.00 & -0.00 & -0.00 & -0.01 \\
\hline 512 & $2.10 \mathrm{e}-5$ & $5.26 \mathrm{e}-6$ & $1.32 \mathrm{e}-6$ & $3.29 \mathrm{e}-7$ & $8.20 \mathrm{e}-8$ \\
\hline
\end{tabular}

TABLE 2: $l_{2}$-norm error and spatial convergence rates for the $\mathrm{CN}$ scheme with various $h$ at $T=0.1$.

\begin{tabular}{|c|c|c|c|c|c|c|c|c|c|}
\hline$N_{t} \backslash N_{x}$ & 100 & rate & 200 & rate & 400 & rate & 800 & rate & 1600 \\
\hline 1 & $3.23 e-5$ & -0.58 & $4.81 \mathrm{e}-5$ & -0.11 & $5.21 \mathrm{e}-5$ & -0.03 & $5.31 \mathrm{e}-5$ & -0.01 & $5.33 e-5$ \\
\hline 2 & $7.73 e-6$ & -0.06 & $8.07 e-6$ & -0.57 & $1.20 \mathrm{e}-5$ & -0.11 & $1.30 \mathrm{e}-5$ & -0.03 & $1.33 e-5$ \\
\hline 4 & $1.77 e-5$ & 3.20 & $1.93 e-6$ & -0.06 & $2.02 \mathrm{e}-6$ & -0.57 & $3.00 \mathrm{e}-6$ & -0.11 & $3.25 e-6$ \\
\hline 8 & $2.02 \mathrm{e}-5$ & 2.19 & $4.43 e-6$ & \begin{tabular}{|l|}
3.20 \\
\end{tabular} & $4.82 \mathrm{e}-7$ & -0.06 & $5.04 \mathrm{e}-7$ & -0.57 & $7.51 \mathrm{e}-7$ \\
\hline 16 & $2.08 \mathrm{e}-5$ & 2.04 & $5.05 e-6$ & \begin{tabular}{|l|}
2.19 \\
\end{tabular} & $1.11 \mathrm{e}-6$ & 3.20 & $1.21 \mathrm{e}-7$ & -0.06 & $1.26 \mathrm{e}-7$ \\
\hline 32 & $2.10 \mathrm{e}-5$ & 2.01 & $5.21 \mathrm{e}-6$ & \begin{tabular}{|l|}
2.04 \\
\end{tabular} & $1.26 \mathrm{e}-6$ & 2.19 & $2.77 \mathrm{e}-7$ & 3.20 & $3.02 \mathrm{e}-8$ \\
\hline 64 & $2.10 e-5$ & 2.00 & $5.25 \mathrm{e}-6$ & \begin{tabular}{|l|}
2.01 \\
\end{tabular} & $1.30 \mathrm{e}-6$ & 2.04 & $3.16 \mathrm{e}-7$ & 2.19 & $6.92 \mathrm{e}-8$ \\
\hline 128 & $2.10 e-5$ & 2.00 & $5.26 \mathrm{e}-6$ & \begin{tabular}{|l|}
2.00 \\
\end{tabular} & $1.31 e-6$ & 2.01 & $3.26 \mathrm{e}-7$ & \begin{tabular}{|l|}
2.04 \\
\end{tabular} & $7.90 \mathrm{e}-8$ \\
\hline 256 & $2.10 e-5$ & 2.00 & $5.26 \mathrm{e}-6$ & \begin{tabular}{|l|}
2.00 \\
\end{tabular} & $1.31 e-6$ & 2.00 & $3.28 \mathrm{e}-7$ & 2.01 & $8.14 \mathrm{e}-8$ \\
\hline 512 & $2.10 \mathrm{e}-5$ & 2.00 & $5.26 \mathrm{e}-6$ & 2.00 & $1.32 \mathrm{e}-6$ & 2.00 & $3.29 \mathrm{e}-7$ & 2.00 & $8.20 \mathrm{e}-8$ \\
\hline
\end{tabular}

From the convergence results in Tables 1, 2, and 3, we can confirm this relation. This convergence relation implies that if we refine both the time and space steps, then we may have the second-order convergence even though one of two convergence rates is not second-order accurate. Interestingly, the convergence relation implies that we do not have the secondorder convergence results for both the spatial and temporal convergence rates at the same starting pair, $\left(N_{x}, N_{t}\right)$.

3.1.2. Convergence Test for the Fully Implicit Scheme. Table 4 lists the $l_{2}$-norm error and temporal convergence rates for the fully implicit scheme with various $\Delta t=T / N_{t}$ and the fixed space step size $h=2 \pi / N_{x}$. As we refine the space grid size, we have more first-order temporal convergence results.

Table 5 lists the $l_{2}$-norm error and spatial convergence rates for the fully implicit scheme with various $h=2 \pi / N_{x}$ and the fixed time step size $\Delta t=T / N_{t}$. As we refine the time step size, we have more second-order spatial convergence results.

Table 6 lists the $l_{2}$-norm error and convergence rates for the fully implicit scheme with various $h=2 \pi / N_{x}$ and $\Delta t=T / N_{t}$. We have a range of convergence results from the first-order to the second-order accuracy. In the lower left triangular region in Table 6, the magnitude of the spatial discretization error dominates that of the temporal discretization error. Therefore, we have the second-order convergence. In the upper right triangular region in Table 6, the magnitude of the temporal discretization error dominates that of the spatial discretization error. Therefore, we have the first-order convergence. This result implies that if we refine both the time and space steps, then we may have the secondorder convergence even though the fully implicit scheme is first-order accurate in temporal discretization. 
TABLE 3: $l_{2}$-norm error and convergence rates for the $\mathrm{CN}$ scheme with various $\Delta t$ and $h$.

\begin{tabular}{|c|c|c|c|c|c|c|c|c|c|}
\hline$N_{t} \backslash N_{x}$ & 100 & rate & 200 & rate & 400 & rate & 800 & rate & 1600 \\
\hline 1 & $3.23 e-5$ & & $4.81 \mathrm{e}-5$ & & $5.21 e-5$ & & $5.31 e-5$ & & $5.33 e-5$ \\
\hline rate & & 2.00 & & 2.00 & & 2.00 & & 2.00 & \\
\hline 2 & $7.73 e-6$ & & $8.07 e-6$ & & $1.20 \mathrm{e}-5$ & & $1.30 e-5$ & & $1.33 e-5$ \\
\hline rate & & 2.00 & & 2.00 & & 2.00 & & 2.00 & \\
\hline 4 & $1.77 \mathrm{e}-5$ & & $1.93 e-6$ & & $2.02 \mathrm{e}-6$ & & $3.00 \mathrm{e}-6$ & & $3.25 \mathrm{e}-6$ \\
\hline rate & & 2.00 & & 2.00 & & 2.00 & & 2.00 & \\
\hline 8 & $2.02 \mathrm{e}-5$ & & $4.43 e-6$ & & $4.82 \mathrm{e}-7$ & & $5.04 \mathrm{e}-7$ & & 7.51e-7 \\
\hline rate & & 2.00 & & 2.00 & & 2.00 & & 2.00 & \\
\hline 16 & $2.08 \mathrm{e}-5$ & & $5.05 e-6$ & & $1.11 \mathrm{e}-6$ & & $1.21 \mathrm{e}-7$ & & $1.26 \mathrm{e}-7$ \\
\hline rate & & 2.00 & & 2.00 & & 2.00 & & 2.00 & \\
\hline 32 & $2.10 \mathrm{e}-5$ & & $5.21 \mathrm{e}-6$ & & $1.26 \mathrm{e}-6$ & & $2.77 \mathrm{e}-7$ & & $3.02 \mathrm{e}-8$ \\
\hline rate & & 2.00 & & 2.00 & & 2.00 & & 2.00 & \\
\hline 64 & $2.10 e-5$ & & $5.25 \mathrm{e}-6$ & & $1.30 \mathrm{e}-6$ & & $3.16 \mathrm{e}-7$ & & $6.92 \mathrm{e}-8$ \\
\hline rate & & 2.00 & & 2.00 & & 2.00 & & 2.00 & \\
\hline 128 & $2.10 \mathrm{e}-5$ & & $5.26 \mathrm{e}-6$ & & $1.31 \mathrm{e}-6$ & & $3.26 \mathrm{e}-7$ & & $7.90 \mathrm{e}-8$ \\
\hline rate & & 2.00 & & 2.00 & & 2.00 & & 2.00 & \\
\hline 256 & $2.10 \mathrm{e}-5$ & & $5.26 \mathrm{e}-6$ & & $1.31 \mathrm{e}-6$ & & $3.28 \mathrm{e}-7$ & & $8.14 \mathrm{e}-8$ \\
\hline rate & & 2.00 & & 2.00 & & 2.00 & & 2.00 & \\
\hline 512 & $2.10 \mathrm{e}-5$ & & $5.26 \mathrm{e}-6$ & & $1.32 \mathrm{e}-6$ & & $3.29 \mathrm{e}-7$ & & $8.20 \mathrm{e}-8$ \\
\hline
\end{tabular}

TABLE $4: l_{2}$-norm error and temporal convergence rates for the fully implicit scheme with various $\Delta t$.

\begin{tabular}{|c|c|c|c|c|c|}
\hline$N_{t} \backslash N_{x}$ & 100 & 200 & 400 & 800 & 1600 \\
\hline 512 & $2.73 e-5$ & $1.15 \mathrm{e}-5$ & $7.56 \mathrm{e}-6$ & $6.58 \mathrm{e}-6$ & $6.33 e-6$ \\
\hline rate & 0.18 & 0.46 & 0.77 & 0.93 & 0.98 \\
\hline 1024 & $2.42 \mathrm{e}-5$ & $8.39 \mathrm{e}-6$ & $4.44 \mathrm{e}-6$ & $3.45 e-6$ & $3.21 \mathrm{e}-6$ \\
\hline rate & 0.10 & 0.30 & 0.63 & 0.87 & 0.96 \\
\hline 2048 & $2.26 e-5$ & $6.82 \mathrm{e}-6$ & $2.88 \mathrm{e}-6$ & $1.89 \mathrm{e}-6$ & $1.64 \mathrm{e}-6$ \\
\hline rate & 0.05 & 0.18 & 0.46 & 0.77 & 0.93 \\
\hline 4096 & $2.18 e-5$ & $6.04 \mathrm{e}-6$ & $2.10 e-6$ & $1.11 e-6$ & $8.63 e-7$ \\
\hline rate & 0.03 & 0.10 & 0.30 & 0.63 & 0.87 \\
\hline 8192 & $2.14 \mathrm{e}-5$ & $5.65 e-6$ & $1.71 \mathrm{e}-6$ & $7.19 \mathrm{e}-7$ & $4.73 e-7$ \\
\hline rate & 0.01 & 0.05 & 0.18 & 0.46 & 0.77 \\
\hline 16384 & $2.12 \mathrm{e}-5$ & $5.46 e-6$ & $1.51 \mathrm{e}-6$ & $5.24 \mathrm{e}-7$ & $2.77 \mathrm{e}-7$ \\
\hline rate & 0.01 & 0.03 & 0.10 & 0.30 & 0.63 \\
\hline 32768 & $2.11 \mathrm{e}-5$ & $5.36 \mathrm{e}-6$ & $1.41 \mathrm{e}-6$ & $4.27 \mathrm{e}-7$ & $1.80 \mathrm{e}-7$ \\
\hline rate & 0.00 & 0.01 & 0.05 & 0.18 & 0.46 \\
\hline 65536 & $2.11 \mathrm{e}-5$ & $5.31 \mathrm{e}-6$ & $1.36 \mathrm{e}-6$ & $3.78 \mathrm{e}-7$ & $1.31 \mathrm{e}-7$ \\
\hline rate & 0.00 & 0.01 & 0.03 & 0.10 & 0.30 \\
\hline 131072 & $2.11 \mathrm{e}-5$ & $5.29 \mathrm{e}-6$ & $1.34 \mathrm{e}-6$ & $3.53 e-7$ & $1.07 \mathrm{e}-7$ \\
\hline rate & 0.00 & 0.00 & 0.01 & 0.05 & 0.18 \\
\hline 262144 & $2.11 \mathrm{e}-5$ & $5.27 \mathrm{e}-6$ & $1.33 e-6$ & $3.41 \mathrm{e}-7$ & $9.44 \mathrm{e}-8$ \\
\hline
\end{tabular}

3.2. The Allen-Cahn Equation. The initial condition is $\phi(x)=$ $0.2 \cos (2 \pi x)$ on $\Omega=(0,1)$. We use $T=1.0 \mathrm{e}-5$, and $\epsilon=0.0075$. Because there is no analytic solution for (7), we consider a reference solution. We define the reference solution $\phi^{\text {ref }}$ as the numerical solution with very fine space and time steps, $\left(\Delta t_{r e f}, h_{r e f}\right)=(3.81 \mathrm{e}-12,1 / 2048)$.
3.2.1. Convergence Test for the CN Scheme. Table 7 lists the $l_{2}$-norm error and temporal convergence rates for the $\mathrm{CN}$ scheme with various $\Delta t=T / N_{t}$ with a fixed space step size $h=1 / 2048$. As we refine the time step size, i.e., $N_{t}=$ $80,160,320$, and 640 , we have the second-order temporal convergence result. 
TABLE 5: $l_{2}$-norm error and spatial convergence rates for the fully implicit scheme with various $h$.

\begin{tabular}{lccccccccc}
\hline$N_{t} \backslash N_{x}$ & 100 & rate & 200 & rate & 400 & rate & 800 & rate & 1600 \\
\hline 512 & $2.73 \mathrm{e}-5$ & 1.25 & $1.15 \mathrm{e}-5$ & 0.61 & $7.56 \mathrm{e}-6$ & 0.20 & $6.58 \mathrm{e}-6$ & 0.06 & $6.33 \mathrm{e}-6$ \\
1024 & $2.42 \mathrm{e}-5$ & 1.53 & $8.39 \mathrm{e}-6$ & 0.92 & $4.44 \mathrm{e}-6$ & 0.36 & $3.45 \mathrm{e}-6$ & 0.11 & $3.21 \mathrm{e}-6$ \\
2048 & $2.26 \mathrm{e}-5$ & 1.73 & $6.82 \mathrm{e}-6$ & 1.25 & $2.88 \mathrm{e}-6$ & 0.61 & $1.89 \mathrm{e}-6$ & 0.20 & $1.64 \mathrm{e}-6$ \\
4096 & $2.18 \mathrm{e}-5$ & 1.85 & $6.04 \mathrm{e}-6$ & 1.53 & $2.10 \mathrm{e}-6$ & 0.92 & $1.11 \mathrm{e}-6$ & 0.36 & $8.63 \mathrm{e}-7$ \\
8192 & $2.14 \mathrm{e}-5$ & 1.92 & $5.65 \mathrm{e}-6$ & 1.73 & $1.71 \mathrm{e}-6$ & 1.25 & $7.19 \mathrm{e}-7$ & 0.61 & $4.73 \mathrm{e}-7$ \\
16384 & $2.12 \mathrm{e}-5$ & 1.96 & $5.46 \mathrm{e}-6$ & 1.85 & $1.51 \mathrm{e}-6$ & 1.53 & $5.24 \mathrm{e}-7$ & 0.92 & $2.77 \mathrm{e}-7$ \\
32768 & $2.11 \mathrm{e}-5$ & 1.98 & $5.36 \mathrm{e}-6$ & 1.92 & $1.41 \mathrm{e}-6$ & 1.73 & $4.27 \mathrm{e}-7$ & 1.25 & $1.80 \mathrm{e}-7$ \\
65536 & $2.11 \mathrm{e}-5$ & 1.99 & $5.31 \mathrm{e}-6$ & 1.96 & $1.36 \mathrm{e}-6$ & 1.85 & $3.78 \mathrm{e}-7$ & 1.53 & $1.31 \mathrm{e}-7$ \\
131072 & $2.11 \mathrm{e}-5$ & 1.99 & $5.29 \mathrm{e}-6$ & 1.98 & $1.34 \mathrm{e}-6$ & 1.92 & $3.53 \mathrm{e}-7$ & 1.73 & $1.07 \mathrm{e}-7$ \\
262144 & $2.11 \mathrm{e}-5$ & 2.00 & $5.27 \mathrm{e}-6$ & 1.99 & $1.33 \mathrm{e}-6$ & 1.96 & $3.41 \mathrm{e}-7$ & 1.85 & $9.44 \mathrm{e}-8$ \\
\hline
\end{tabular}

TABLE 6: $l_{2}$-norm error and convergence rates for the fully implicit scheme with various $\Delta t$ and $h$.

\begin{tabular}{|c|c|c|c|c|c|c|c|c|c|}
\hline$\overline{N_{t} \backslash N_{x}}$ & 100 & rate & 200 & rate & 400 & rate & 800 & rate & 1600 \\
\hline 512 & $2.73 e-5$ & & $1.15 \mathrm{e}-5$ & & $7.56 e-6$ & & $6.58 \mathrm{e}-6$ & & $6.33 e-6$ \\
\hline rate & & 1.70 & & 1.37 & & 1.13 & & 1.04 & \\
\hline 1024 & $2.42 \mathrm{e}-5$ & & $8.39 \mathrm{e}-6$ & & $4.44 \mathrm{e}-6$ & & $3.45 e-6$ & & $3.21 \mathrm{e}-6$ \\
\hline rate & & 1.82 & & 1.54 & & 1.23 & & 1.07 & \\
\hline 2048 & $2.26 e-5$ & & $6.82 \mathrm{e}-6$ & & $2.88 \mathrm{e}-6$ & & $1.89 e-6$ & & $1.64 \mathrm{e}-6$ \\
\hline rate & & 1.90 & & 1.70 & & 1.37 & & 1.13 & \\
\hline 4096 & $2.18 \mathrm{e}-5$ & & $6.04 \mathrm{e}-6$ & & $2.10 \mathrm{e}-6$ & & $1.11 \mathrm{e}-6$ & & $8.63 e-7$ \\
\hline rate & & 1.95 & & 1.82 & & 1.54 & & 1.23 & \\
\hline 8192 & $2.14 \mathrm{e}-5$ & & $5.65 e-6$ & & $1.71 e-6$ & & $7.19 \mathrm{e}-7$ & & $4.73 e-7$ \\
\hline rate & & 1.97 & & 1.90 & & 1.70 & & 1.37 & \\
\hline 16384 & $2.12 \mathrm{e}-5$ & & $5.46 \mathrm{e}-6$ & & $1.51 \mathrm{e}-6$ & & $5.24 \mathrm{e}-7$ & & $2.77 \mathrm{e}-7$ \\
\hline rate & & 1.99 & & 1.95 & & 1.82 & & 1.54 & \\
\hline 32768 & $2.11 e-5$ & & $5.36 e-6$ & & $1.41 \mathrm{e}-6$ & & $4.27 \mathrm{e}-7$ & & $1.80 \mathrm{e}-7$ \\
\hline rate & & \begin{tabular}{|l|}
1.99 \\
\end{tabular} & & 1.97 & & 1.90 & & 1.70 & \\
\hline 65536 & $2.11 e-5$ & & $5.31 \mathrm{e}-6$ & & $1.36 \mathrm{e}-6$ & & $3.78 \mathrm{e}-7$ & & $1.31 \mathrm{e}-7$ \\
\hline rate & & 2.00 & & 1.99 & & 1.95 & & 1.82 & \\
\hline 131072 & $2.11 e-5$ & & $5.29 \mathrm{e}-6$ & & $1.34 \mathrm{e}-6$ & & $3.53 \mathrm{e}-7$ & & $1.07 \mathrm{e}-7$ \\
\hline rate & & 2.00 & & \begin{tabular}{|l|}
1.99 \\
\end{tabular} & & 1.97 & & 1.90 & \\
\hline 262144 & $2.11 \mathrm{e}-5$ & & $5.27 \mathrm{e}-6$ & & $1.33 e-6$ & & $3.41 \mathrm{e}-7$ & & $9.44 \mathrm{e}-8$ \\
\hline
\end{tabular}

TABLE 7: $l_{2}$-norm error and temporal convergence rates for the CN scheme with various $\Delta t .(\Delta t, h)=(1.25 \mathrm{e}-7,1 / 2048)$ and $\left(\Delta t_{r e f}, h_{r e f}\right)=$ (3.81e-12, 1/2048) are used.

\begin{tabular}{llllllll}
\hline case & $(\Delta t, h)$ & rate & $(\Delta t / 2, h)$ & rate & $(\Delta t / 4, h)$ & rate & $(\Delta t / 8, h)$ \\
\hline$l_{2}$-error & $7.13 \mathrm{e}-9$ & 2.00 & $1.78 \mathrm{e}-9$ & 2.00 & $4.46 \mathrm{e}-10$ & 2.00 & $1.11 \mathrm{e}-10$ \\
\hline
\end{tabular}

TABLE 8: $l_{2}$-norm error and spatial convergence rates for the CN scheme with various $h$. $(\Delta t, h)=(3.81 \mathrm{e}-12,1 / 8)$ and $\left(\Delta t_{r e f}, h_{r e f}\right)=(3.81 \mathrm{e}-12$, $1 / 2048$ ) are used.

\begin{tabular}{lccccccc}
\hline case & $(\Delta t, h)$ & rate & $(\Delta t, h / 2)$ & rate & $(\Delta t, h / 4)$ & rate & $(\Delta t, h / 8)$ \\
\hline$l_{2}$-error & $3.45 \mathrm{e}-6$ & 2.00 & $8.61 \mathrm{e}-7$ & 2.01 & $2.14 \mathrm{e}-7$ & 2.01 & $5.32 \mathrm{e}-8$ \\
\hline
\end{tabular}

Table 8 lists the $l_{2}$-norm error and spatial convergence rates for the $\mathrm{CN}$ scheme with various $h=1 / N_{x}$ with a fixed time step size $\Delta t=T / 2621440$. As we refine the space grid size, i.e., $N_{x}=8,16,32$ and 64 , we have the second-order spatial convergence result.
Tables 9 and 10 list the $l_{2}$-norm error and spatial and temporal convergence rates for the $\mathrm{CN}$ scheme with various $h=1 / N_{x}$ and $\Delta t=T / N_{t}$. The results generate the secondorder convergence for both the cases, i.e., $(\Delta t, h)=(1.25 \mathrm{e}-7$, $1 / 64)$ and $(\Delta t, h)=(6.10 \mathrm{e}-11,1 / 8)$. 
TABLE 9: $l_{2}$-norm error and convergence rates for the CN scheme with various $h$ and $\Delta t .(\Delta t, h)=(1.25 \mathrm{e}-7,1 / 64)$ and $\left(\Delta t_{r e f}, h_{r e f}\right)=(3.81 \mathrm{e}-12$, $1 / 2048$ ) are used.

\begin{tabular}{lllccccc}
\hline case & $(\Delta t, h)$ & rate & $(\Delta t / 2, h / 2)$ & rate & $(\Delta t / 4, h / 4)$ & rate & $(\Delta t / 8, h / 8)$ \\
\hline$l_{2}$-error & $6.03 \mathrm{e}-8$ & 2.01 & $1.50 \mathrm{e}-8$ & 2.02 & $3.69 \mathrm{e}-9$ & 2.09 & $8.68 \mathrm{e}-10$ \\
\hline
\end{tabular}

TABLE 10: $l_{2}$-norm error and convergence rates for the CN scheme with various $h$ and $\Delta t .(\Delta t, h)=(6.10 \mathrm{e}-11,1 / 8)$ and $\left(\Delta t_{r e f}, h_{r e f}\right)=(3.81 \mathrm{e}-12$, $1 / 2048$ ) are used.

\begin{tabular}{lccccccc}
\hline case & $(\Delta t, h)$ & rate & $(\Delta t / 2, h / 2)$ & rate & $(\Delta t / 4, h / 4)$ & rate & $(\Delta t / 8, h / 8)$ \\
\hline$l_{2}$-error & $3.45 \mathrm{e}-6$ & 2.00 & $8.61 \mathrm{e}-7$ & 2.01 & $2.14 \mathrm{e}-7$ & 2.01 & $5.32 \mathrm{e}-8$ \\
\hline
\end{tabular}

TABLE $11: l_{2}$-norm error and temporal convergence rates for the fully implicit scheme with various $\Delta t .(\Delta t, h)=(1.25 \mathrm{e}-07,1 / 2048)$ and $\left(\Delta t_{r e f}\right.$, $\left.h_{r e f}\right)=(3.81 \mathrm{e}-12,1 / 2048)$ are used.

\begin{tabular}{lccccccc}
\hline case & $(\Delta t, h)$ & rate & $(\Delta t / 2, h)$ & rate & $(\Delta t / 4, h)$ & rate & $(\Delta t / 8, h)$ \\
\hline$l_{2}$-error & $2.82 \mathrm{e}-5$ & 1.01 & $1.40 \mathrm{e}-5$ & 1.01 & $6.96 \mathrm{e}-6$ & 1.02 & $3.42 \mathrm{e}-6$ \\
\hline
\end{tabular}

TABLE 12: $l_{2}$-norm error and spatial convergence rates for the fully implicit scheme with various $h .(\Delta t, h)=(3.81 \mathrm{e}-12,1 / 8)$ and $\left(\Delta t_{r e f}, h_{r e f}\right)=$ (3.81e-12, 1/2048) are used.

\begin{tabular}{lccccccc}
\hline case & $(\Delta t, h)$ & rate & $(\Delta t, h / 2)$ & rate & $(\Delta t, h / 4)$ & rate & $(\Delta t, h / 8)$ \\
\hline$l_{2}$-error & $3.46 \mathrm{e}-6$ & 1.99 & $8.69 \mathrm{e}-7$ & 1.97 & $2.22 \mathrm{e}-7$ & 1.86 & $6.10 \mathrm{e}-8$ \\
\hline
\end{tabular}

TABLE 13: $l_{2}$-norm error and convergence rates for the fully implicit scheme with various $\Delta t$ and $h .(\Delta t, h)=(1.25 \mathrm{e}-7,1 / 64)$ and $\left(\Delta t_{r e f}, h_{r e f}\right)=$ (3.81e-12, 1/2048) are used.

\begin{tabular}{lccccccc}
\hline case & $(\Delta t, h)$ & rate & $(\Delta t / 2, h / 2)$ & rate & $(\Delta t / 4, h / 4)$ & rate & $(\Delta t / 8, h / 8)$ \\
\hline$l_{2}$-error & $2.85 \mathrm{e}-5$ & 1.01 & $1.42 \mathrm{e}-5$ & 1.00 & $7.08 \mathrm{e}-6$ & 1.00 & $3.54 \mathrm{e}-6$ \\
\hline
\end{tabular}

TABLE $14: l_{2}$-norm error and convergence rates for the fully implicit scheme with various $\Delta t$ and $h .(\Delta t, h)=(6.10 \mathrm{e}-11,1 / 8)$ and $\left(\Delta t_{r e f}, h_{r e f}\right)=$ $(3.81 \mathrm{e}-12,1 / 2048)$ are used.

\begin{tabular}{lllccccc}
\hline case & $(\Delta t, h)$ & rate & $(\Delta t / 2, h / 2)$ & rate & $(\Delta t / 4, h / 4)$ & rate & $(\Delta t / 8, h / 8)$ \\
\hline$l_{2}$-error & $3.47 \mathrm{e}-6$ & 2.00 & $8.67 \mathrm{e}-7$ & 2.00 & $2.17 \mathrm{e}-7$ & 2.00 & $5.41 \mathrm{e}-8$ \\
\hline
\end{tabular}

3.2.2. Convergence Test for the Fully Implicit Scheme. Table 11 lists the $l_{2}$-norm error and temporal convergence rates for the fully implicit scheme with various $\Delta t=T / N_{t}$ and a fixed space step size $h=1 / 2048$. As we refine the time step size, i.e., $N_{t}=80,160,320$, and 640 , we have the first-order temporal convergence result.

Table 12 lists the $l_{2}$-norm error and spatial convergence rates for the fully implicit scheme with various $h=1 / 2048$ with a fixed time step size $\Delta t=T / N_{t}$. As we refine the space grid size, i.e., $N_{x}=8,16,32$, and 64 , we have the second-order spatial convergence result.

Tables 13 and 14 list the $l_{2}$-norm error and spatial and temporal convergence rates for the fully implicit scheme with various $h=1 / N_{x}$ and $\Delta t=T / N_{t}$, respectively.

Table 13 shows that the convergence rate appears to be only first-order with $N_{t}=80$ and $N_{x}=64$. On the other hand, Table 14 shows that the convergence rate appears to be second-order with $N_{t}=163840$ and $N_{x}=8$. These results demonstrate that even if the numerical scheme is only first-order in time and second-order in space, we may have the second-order convergence with some refinement combination.

3.3. The Cahn-Hilliard Equation. The initial condition is $\phi(x)=0.1 \cos (2 \pi x)$ on $\Omega=(0,1)$. Table 15 lists the $l_{2}$-norm error and temporal convergence rates for the unconditionally stable scheme with various $\Delta t=T / N_{t}$ with a fixed space step size $h=1 / N_{x}$. We use $N_{x}=1024, T=2.40 \mathrm{e}-3$, and $\epsilon=0.02$. We define the reference solution with fine time step $\Delta t=9.54 \mathrm{e}-6$. We find that the convergence rates are all firstorder.

Table 16 lists the $l_{2}$-norm error and spatial convergence rates with various $h=1 / N_{x}$ with a fixed time step size $\Delta t=$ $T / N_{t}$. We use $\Delta t=9.54 \mathrm{e}-6, T=2.40 \mathrm{e}-3$, and $\epsilon=0.02$. We define the reference solution with fine space step $h=1 / 1024$. We can find that the convergence rates are second-order.

Table 17 lists the $l_{2}$-norm error and spatial and temporal convergence rates with various $h=1 / N_{x}$ and $\Delta t$. We use $T=$ 
TABLE 15: $l_{2}$-norm error and temporal convergence rates for the unconditionally stable numerical scheme with various $\Delta t$. $(\Delta t, h)=(3.05 \mathrm{e}-4$, $1 / 1024)$ and $\left(\Delta t_{r e f}, h_{r e f}\right)=(9.54 \mathrm{e}-6,1 / 1024)$ are used.

\begin{tabular}{lccccc}
\hline case & $(\Delta t, h)$ & rate & $(\Delta t / 2, h)$ & rate & $(\Delta t / 4, h)$ \\
\hline$l_{2}$-error & $4.71 \mathrm{e}-5$ & 1.05 & $2.27 \mathrm{e}-5$ & 1.11 & $1.05 \mathrm{e}-5$ \\
\hline
\end{tabular}

TABLE $16: l_{2}$-norm error and spatial convergence rates for the unconditionally stable numerical scheme with various $h .(\Delta t, h)=(9.54 \mathrm{e}-6,1 / 16)$ and $\left(\Delta t_{r e f}, h_{r e f}\right)=(9.54 \mathrm{e}-6,1 / 1024)$ are used.

\begin{tabular}{llcccc}
\hline case & $(\Delta t, h)$ & rate & $(\Delta t, h / 2)$ & rate & $(\Delta t, h / 4)$ \\
\hline$l_{2}$-error & $9.90 \mathrm{e}-5$ & 1.98 & $2.52 \mathrm{e}-5$ & 1.96 & $6.46 \mathrm{e}-6$ \\
\hline
\end{tabular}

TABLE $17: l_{2}$-norm error and convergence rates for the unconditionally stable numerical scheme with various $\Delta t$ and $h .(\Delta t, h)=(1.20 \mathrm{e}-3,1 / 32)$ and $\left(\Delta t_{r e f}, h_{r e f}\right)=(9.54 \mathrm{e}-6,1 / 1024)$ are used.

\begin{tabular}{lccccc}
\hline case & $(\Delta t, h)$ & rate & $(\Delta t / 2, h / 2)$ & rate & $(\Delta t / 4, h / 4)$ \\
\hline$l_{2}$-error & $2.06 \mathrm{e}-4$ & 1.06 & $9.87 \mathrm{e}-5$ & 1.00 & $4.93 \mathrm{e}-5$ \\
\hline
\end{tabular}

TABLE 18: $l_{2}$-norm error and convergence rates for the unconditionally stable numerical scheme with various $\Delta t$ and $h .(\Delta t, h)=(7.63 \mathrm{e}-5,1 / 8)$ and $\left(\Delta t_{r e f}, h_{r e f}\right)=(9.54 \mathrm{e}-6,1 / 1024)$ are used.

\begin{tabular}{lccccc}
\hline case & $(\Delta t, h)$ & rate & $(\Delta t / 2, h / 2)$ & rate & $(\Delta t / 4, h / 4)$ \\
\hline$l_{2}$-error & $3.88 \mathrm{e}-4$ & 1.91 & $1.03 \mathrm{e}-4$ & 1.95 & $2.67 \mathrm{e}-5$ \\
\hline
\end{tabular}

$2.40 \mathrm{e}-3$ and $\epsilon=0.02$. We can find that the convergence rates are first-order.

Table 18 lists the $l_{2}$-norm error and spatial and temporal convergence rates with various $h=1 / N_{x}$ and $\Delta t$. We use $T=$ $2.40 \mathrm{e}-3$ and $\epsilon=0.02$. We can find that the convergence rates are second-order.

The results in Tables 17 and 18 show that we may have the first-order or the second-order convergence rates depending on starting spatial and temporal step sizes in the case of the first-order in time and the second-order in space scheme.

\section{Conclusion}

We presented verification methods for the convergence rates of the numerical solutions for parabolic equations. As examples, we considered the numerical convergence rates of the heat equation, the $\mathrm{AC}$ equation, and the $\mathrm{CH}$ equation. Convergence test results showed that if we refine the spatial and temporal steps at the same time, then we may have the second-order convergence rate for the fully implicit scheme, which is first-order accurate in time and secondorder accurate in space. Therefore, for a rigorous numerical convergence test, we need to perform the spatial and the temporal convergence tests separately.

\section{Data Availability}

The data used to support the findings of this study are available from the corresponding author upon request.

\section{Conflicts of Interest}

The authors declare that there are no conflicts of interest regarding the publication of this paper.

\section{Acknowledgments}

The first author (D. Jeong) was supported by the National Research Foundation of Korea (NRF) grant funded by the Korea government (MSIP) (NRF-2017R1E1A1A03070953). The corresponding author (J.S. Kim) was supported by Korea University Future Research Grant. The authors (C. Lee and J. Yang) express their thanks for the support from the BK21 PLUS program.

\section{References}

[1] D. Han and X. Wang, "A second order in time, uniquely solvable, unconditionally stable numerical scheme for Cahn-HilliardNavier-Stokes equation," Journal of Computational Physics, vol. 290, pp. 139-156, 2015.

[2] W. Y. Tian, H. Zhou, and W. H. Deng, "A class of second order difference approximations for solving space fractional diffusion equations," Mathematics of Computation, vol. 84, no. 294, pp. 1703-1727, 2015.

[3] L. B. Feng, P. Zhuang, F. Liu, I. Turner, V. Anh, and J. Li, "A fast second-order accurate method for a two-sided space-fractional diffusion equation with variable coefficients," Computers \& Mathematics with Applications, vol. 73, no. 6, pp. 1155-1171, 2017.

[4] X. Wu, G. J. van Zwieten, and K. G. van der Zee, "Stabilized second-order convex splitting schemes for Cahn-Hilliard models with application to diffuse-interface tumor-growth models," International Journal for Numerical Methods in Biomedical Engineering, vol. 30, no. 2, pp. 180-203, 2014.

[5] R. K. Jaiman, N. R. Pillalamarri, and M. Z. Guan, "A stable second-order partitioned iterative scheme for freely vibrating low-mass bluff bodies in a uniform flow," Computer Methods Applied Mechanics and Engineering, vol. 301, pp. 187-215, 2016. 
[6] K. Sagiyama, S. Rudraraju, and K. Garikipati, "Unconditionally stable, second-order accurate schemes for solid state phase transformations driven by mechano-chemical spinodal decomposition," Computer Methods Applied Mechanics and Engineering, vol. 311, pp. 556-575, 2016.

[7] J. Cao, Y. Qiu, and G. Song, "A compact finite difference scheme for variable order subdiffusion equation," Communications in Nonlinear Science and Numerical Simulation, vol. 48, pp. 140149, 2017.

[8] L. Pinto and E. Sousa, "Numerical solution of a time-space fractional Fokker Planck equation with variable force field and diffusion," Communications in Nonlinear Science and Numerical Simulation, vol. 50, pp. 211-228, 2017.

[9] Y. Li, J. Kim, and N. Wang, "An unconditionally energy-stable second-order time-accurate scheme for the Cahn-Hilliard equation on surfaces," Communications in Nonlinear Science and Numerical Simulation, vol. 53, pp. 213-227, 2017.

[10] D. Jeong, Y. Choi, and J. Kim, "A benchmark problem for the two- and three-dimensional Cahn-Hilliard equations," Communications in Nonlinear Science and Numerical Simulation, vol. 61, pp. 149-159, 2018.

[11] Y. Choi, D. Jeong, and J. Kim, "A multigrid solution for the Cahn-Hilliard equation on nonuniform grids," Applied Mathematics and Computation, vol. 293, pp. 320-333, 2017.

[12] M. Ran, T. Luo, and L. Zhang, "Unconditionally stable compact theta schemes for solving the linear and semi-linear fourthorder diffusion equations," Applied Mathematics and Computation, vol. 342, pp. 118-129, 2019.

[13] A. Voit, A. Krekhov, W. Enge, L. Kramer, and W. Köhler, "Thermal patterning of a critical polymer blend," Physical Review Letters, vol. 94, no. 21, 2005.

[14] G. Deugoue and T. Tachim Medjo, "Convergence of the solution of the stochastic 3D globally modified Cahn-Hilliard-NavierStokes equations," Journal of Differential Equations, vol. 265, no. 2, pp. 545-592, 2018.

[15] D. C. Antonopoulou, D. Farazakis, and G. Karali, "Malliavin calculus for the stochastic CAHn-Hilliard/Allen-CAHn equation with unbounded noise diffusion," Journal of Differential Equations, vol. 265, no. 7, pp. 3168-3211, 2018.

[16] K. Kuto, T. Mori, T. Tsujikawa, and S. Yotsutani, "Secondary bifurcation for a nonlocal Allen-Cahn equation," Journal of Differential Equations, vol. 263, no. 5, pp. 2687-2714, 2017.

[17] K. D. Phung, G. Wang, and Y. Xu, "Impulse output rapid stabilization for heat equations," Journal of Differential Equations, vol. 263, no. 8, pp. 5012-5041, 2017.

[18] S. Lee and J. Shin, "Energy stable compact scheme for CahnHilliard equation with periodic boundary condition," Computers \& Mathematics with Applications, vol. 77, no. 1, pp. 189-198, 2018.

[19] X. Yang, J. Zhao, and X. He, "Linear, second order and unconditionally energy stable schemes for the viscous CahnHilliard equation with hyperbolic relaxation using the invariant energy quadratization method," Journal of Computational and Applied Mathematics, vol. 343, pp. 80-97, 2018.

[20] K. Cheng, W. Feng, C. Wang, and S. M. Wise, "An energy stable fourth order finite difference scheme for the Cahn-Hilliard equation," Journal of Computational and Applied Mathematics, vol. 51, no. 1, 2018.

[21] A. Horvat, G. J. A. Sevink, A. V. Zvelindovsky, A. Krekhov, and L. Tsarkova, "Specific features of defect structure and dynamics in the cylinder phase of block copolymers," ACS Nano, vol. 2, no. 6, pp. 1143-1152, 2008.
[22] S. M. Allen and J. W. Cahn, "A microscopic theory for antiphase boundary motion and its application to antiphase domain coarsening," Acta Metallurgica et Materialia, vol. 27, no. 6, pp. 1085-1095, 1979.

[23] D. Lee and S. Lee, "Image segmentation based on modified fractional Allen-Cahn equation," Mathematical Problems in Engineering, vol. 2019, no. 5, Article ID 3980181, 6 pages, 2019.

[24] D. S. Lee and J. S. Kim, "Mean curvature flow by the Allen-Cahn equation," European Journal of Applied Mathematics, vol. 26, no. 4, pp. 535-559, 2015.

[25] X. Feng and A. Prohl, "Numerical analysis of the AllenCahn equation and approximation for mean curvature flows," Numerische Mathematik, vol. 94, no. 1, pp. 33-65, 2003.

[26] X. Feng and Y. Li, "Analysis of symmetric interior penalty discontinuous Galerkin methods for the Allen-Cahn equation and the mean curvature flow," IMA Journal of Numerical Analysis (IMAJNA), vol. 35, no. 4, pp. 1622-1651, 2015.

[27] L. Ma, R. Chen, X. Yang, and H. Zhang, "Numerical approximations for Allen-Cahn type phase field model of two-phase incompressible fluids with moving contact lines," Communications in Computational Physics, vol. 21, no. 3, pp. 867-889, 2017.

[28] T. Takaki, T. Shimokawabe, M. Ohno, A. Yamanaka, and T. Aoki, "Unexpected selection of growing dendrites by verylarge-scale phase-field simulation," Journal of Crystal Growth, vol. 382, pp. 21-25, 2013.

[29] J. Zhao, Q. Wang, and X. Yang, "Numerical approximations for a phase field dendritic crystal growth model based on the invariant energy quadratization approach," International Journal for Numerical Methods in Engineering, vol. 110, no. 3, pp. 279-300, 2017.

[30] Y. Li, D. Jeong, J.-I. Choi, S. Lee, and J. Kim, "Fast local image inpainting based on the Allen-Cahn model," Digital Signal Processing, vol. 37, no. 1, pp. 65-74, 2015.

[31] M. Beneš, V. r. Chalupecky, and K. Mikula, "Geometrical image segmentation by the Allen-Cahn equation," Applied Numerical Mathematics, vol. 51, no. 2-3, pp. 187-205, 2004.

[32] Y. Li and J. Kim, "An unconditionally stable hybrid method for image segmentation," Applied Numerical Mathematics, vol. 82, pp. 32-43, 2014.

[33] J. W. Cahn and J. E. Hilliard, "Free energy of a nonuniform system. I. Interfacial free energy," The Journal of Chemical Physics, vol. 28, no. 2, pp. 258-267, 1958.

[34] M. Maraldi, L. Molari, and D. Grandi, "A unified thermodynamic framework for the modelling of diffusive and displacive phase transitions," International Journal of Engineering Science, vol. 50, no. 1, pp. 31-45, 2012.

[35] S. Zhou and M. Y. Wang, "Multimaterial structural topology optimization with a generalized Cahn-Hilliard model of multiphase transition," Structural and Multidisciplinary Optimization, vol. 33, no. 2, pp. 89-111, 2007.

[36] V. E. Badalassi, H. D. Ceniceros, and S. Banerjee, "Computation of multiphase systems with phase field models," Journal of Computational Physics, vol. 190, no. 2, pp. 371-397, 2003.

[37] Y. Li, J.-I. Choi, and J. Kim, "Multi-component CahnHilliard system with different boundary conditions in complex domains," Journal of Computational Physics, vol. 323, pp. 1-16, 2016.

[38] Y. Li, J.-I. Choi, and J. Kim, "A phase-field fluid modeling and computation with interfacial profile correction term," Communications in Nonlinear Science and Numerical Simulation, vol. 30, no. 1-3, pp. 84-100, 2016. 
[39] M. Kotschote and R. Zacher, "Strong solutions in the dynamical theory of compressible fluid mixtures," Mathematical Models and Methods in Applied Sciences, vol. 25, no. 7, pp. 1217-1256, 2015.

[40] A. L. Bertozzi, S. Esedoglu, and A. Gillette, "Inpainting of binary images using the Cahn-Hilliard equation," IEEE Transactions on Image Processing, vol. 16, no. 1, pp. 285-291, 2007.

[41] Y. Li, J. Shin, Y. Choi, and J. Kim, "Three-dimensional volume reconstruction from slice data using phase-field models," Computer Vision and Image Understanding, vol. 137, pp. 115-124, 2015.

[42] D. Jeong, J. Shin, Y. Li et al., "Numerical analysis of energyminimizing wavelengths of equilibrium states for diblock copolymers," Current Applied Physics, vol. 14, no. 9, pp. 12631272, 2014.

[43] S. M. Wise, J. S. Lowengrub, H. B. Frieboes, and V. Cristini, "Three-dimensional multispecies nonlinear tumor growth? I: model and numerical method," Journal of Theoretical Biology, vol. 253, no. 3, pp. 524-543, 2008.

[44] J. Zhu, L.-Q. Chen, and J. Shen, "Morphological evolution during phase separation and coarsening with strong inhomogeneous elasticity," Modelling and Simulation in Materials Science and Engineering, vol. 9, no. 6, pp. 499-511, 2001.

[45] J. D. Faires and R. L. Burden, Numerical Analysis, Cengage Learning, Calif, USA, 9th edition, 2011.

[46] L. B. Feng, P. Zhuang, F. Liu, I. Turner, and J. Li, "High-order numerical methods for the Riesz space fractional advectiondispersion equations," Computers \& Mathematics with Applications Available online, vol. 19, 2016.

[47] U. Trottenberg, C. W. Oosterlee, and A. schuller, Multigrid, Academic Press, London, UK, 2001.

[48] J. Kim, "A numerical method for the Cahn-Hilliard equation with a variable mobility," Communications in Nonlinear Science and Numerical Simulation, vol. 12, no. 8, pp. 1560-1571, 2007.

[49] Y. Wang and Y. Ge, "High-Order compact difference scheme and multigrid method for solving the 2D elliptic problems," Mathematical Problems in Engineering, vol. 2018, Article ID 7831731, 11 pages, 2018.

[50] D. J. Eyre, Computational and Mathematical Models of Microstructural Evolution, The Material Research Society, Warrendale, PA, USA, 1998. 


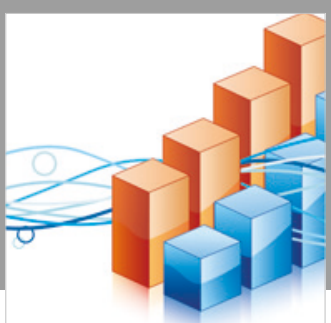

Advances in

Operations Research

\section{-n-m}
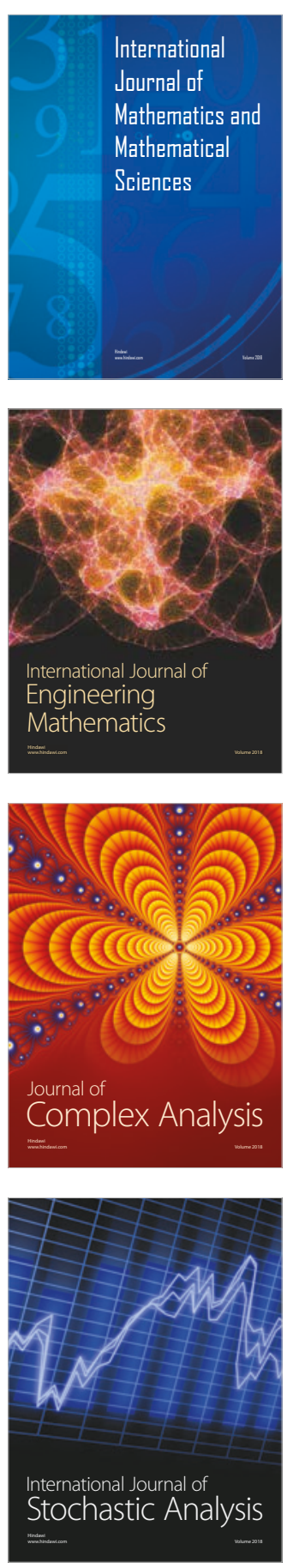
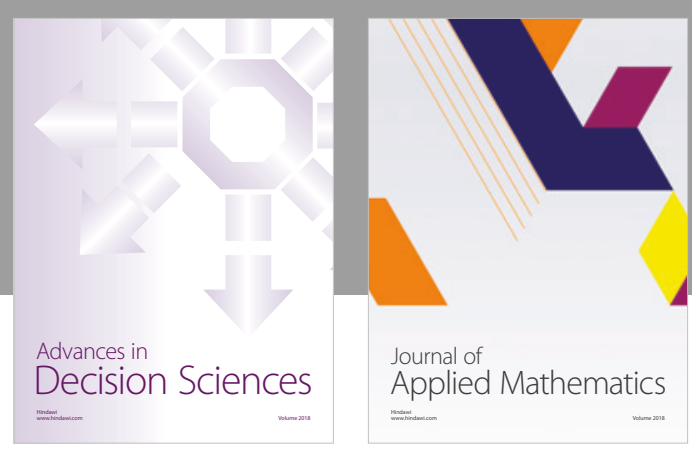

Journal of

Applied Mathematics
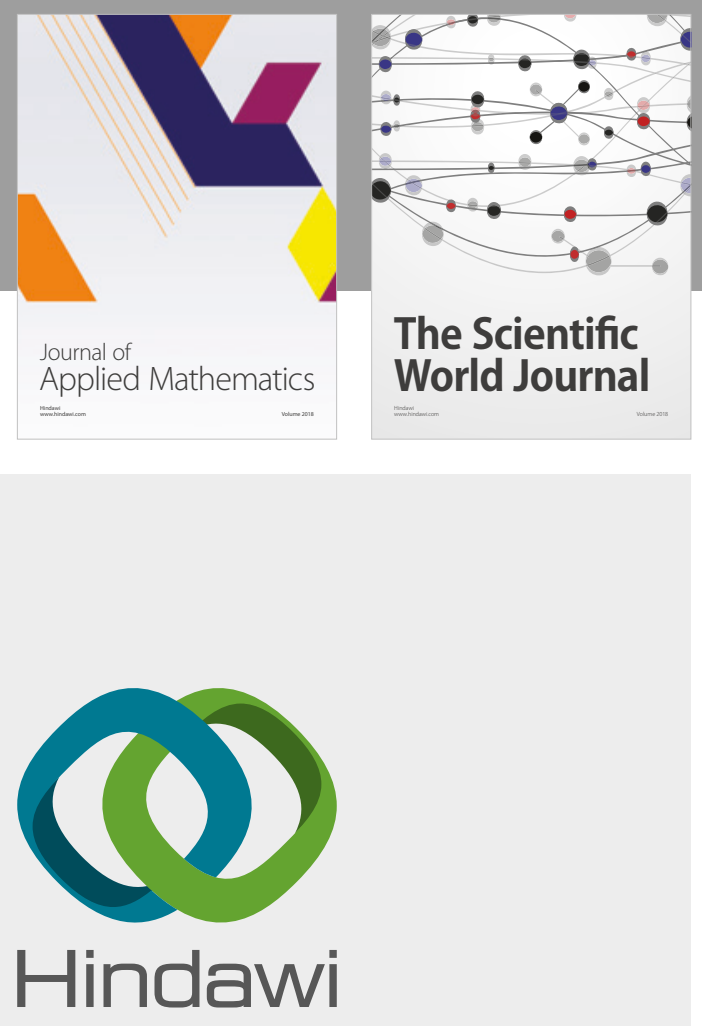

Submit your manuscripts at

www.hindawi.com

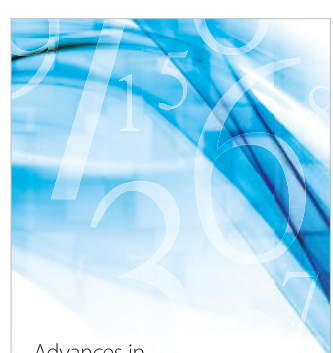

Advances in
Numerical Analysis
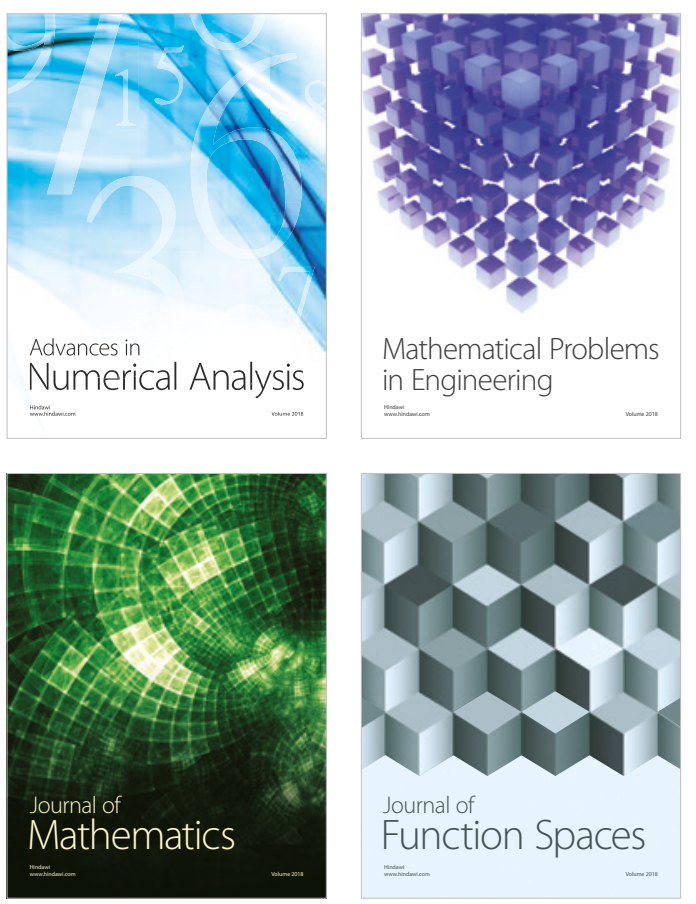

Mathematical Problems in Engineering

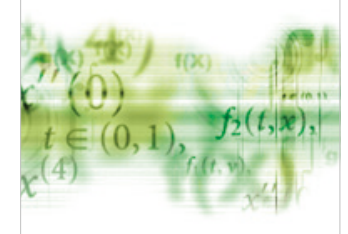

International Journal of

Differential Equations

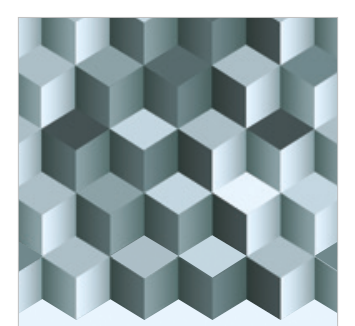

Journal of

Function Spaces
The Scientific

World Journal

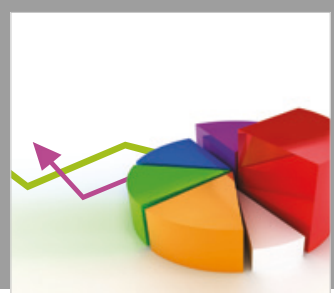

Journal of

Probability and Statistics
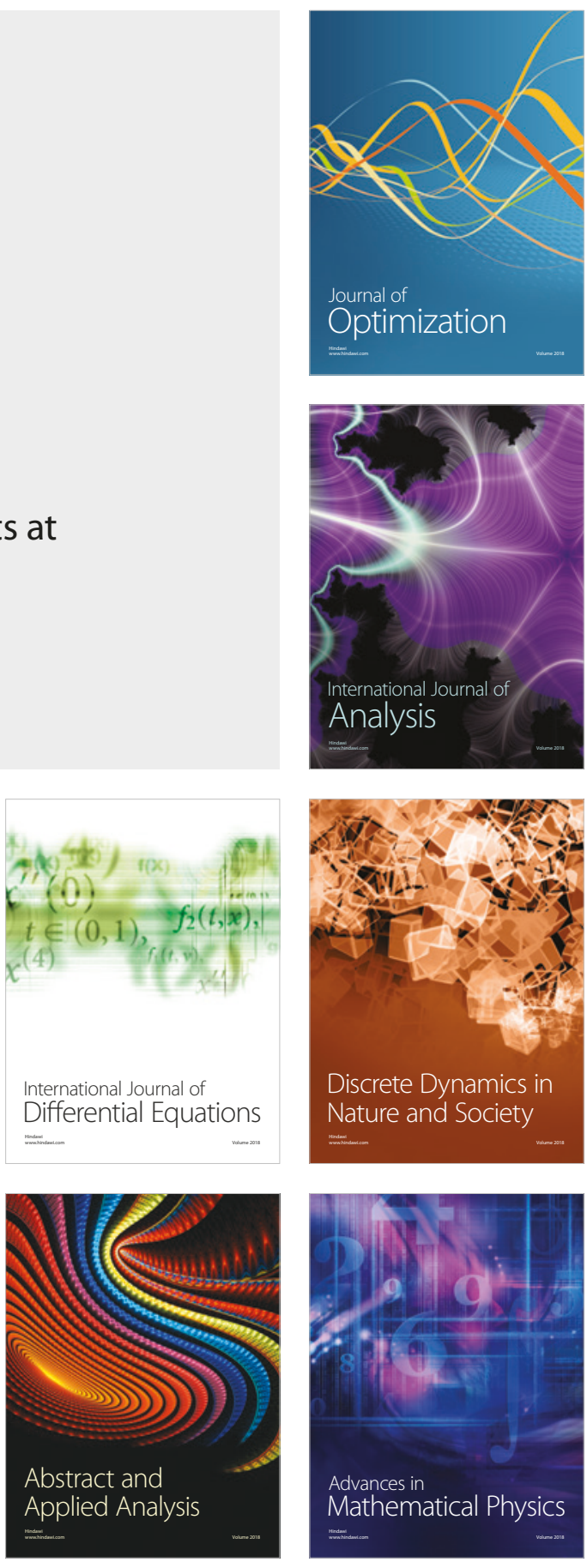